CORRECTION

https://doi.org/10.1038/s41586-019-1108-4

\title{
Author Correction: Consistent \\ success in life-supporting porcine cardiac xenotransplantation
}

Matthias Längin, Tanja Mayr, Bruno Reichart,

Sebastian Michel, Stefan Buchholz, Sonja Guethoff, Alexey Dashkevich, Andrea Baehr, Stefanie Egerer, Andreas Bauer, Maks Mihalj, Alessandro Panelli, Lara Issl, Jiawei Ying, Ann Kathrin Fresch, Ines Buttgereit, Maren Mokelke, Julia Radan, Fabian Werner, Isabelle Lutzmann, Stig Steen, Trygve Sjöberg, Audrius Paskevicius, Liao Qiuming, Riccardo Sfriso, Robert Rieben, Maik Dahlhoff, Barbara Kessler, Elisabeth Kemter, Mayuko Kurome, Valeri Zakhartchenko, Katharina Klett, Rabea Hinkel, Christian Kupatt,

Almuth Falkenau, Simone Reu, Reinhard Ellgass, Rudolf Herzog, Uli Binder, Günter Wich, Arne Skerra, David Ayares, Alexander Kind, Uwe Schönmann, Franz-Josef Kaup, Christian Hagl, Eckhard Wolf, Nikolai Klymiuk, Paolo Brenner \& Jan-Michael Abicht

Correction to: Nature https://doi.org/10.1038/s41586-018-0765-z, published online 05 December 2018.

In this Letter, Mayuko Kurome and Valeri Zakhartchenko should have been listed as authors, with the affiliation: Institute of Molecular Animal Breeding and Biotechnology, Gene Center, LMU Munich, Munich, Germany. Both authors contributed to this work by providing genetically multi-modified donor pigs (see 'Author contributions'). The Letter has been corrected online. 